\title{
Treatment of Long-Limb Biliary-Enteric Anastomotic Strictures: ERCP, PTBD, or EUS?
}

\author{
Yousuke Nakai ${ }^{1}$ \\ Published online: 4 July 2019 \\ ○) Springer Science+Business Media, LLC, part of Springer Nature 2019
}

Benign biliary strictures following bile duct injury or liver transplantation are often refractory to non-surgical treatments such as endoscopic retrograde cholangiopancreatography (ERCP) or percutaneous transhepatic biliary drainage (PTBD) [1]. Endoscopic stricture dilation with or without stent placement is now the standard of care due to its lesser invasiveness [2]. Since in cases with a long-limb Roux-en-Y reconstruction, access to the biliary-enteric anastomosis is technically difficult or even impossible with conventional endoscopes, PTBD has assumed importance in this setting. Recent development of device-assisted enteroscopy [3], especially short-type enteroscopy [4], facilitates endoscopic biliary access as well as therapeutic interventions in patients with surgically altered anatomy.

In this issue of Digestive Diseases and Sciences, Hammad et al. [5] conducted a retrospective comparative study of enteroscopy-assisted ERCP vs. PTBD for the management of the biliary-enteric anastomotic stricture. The studies reported comparable outcomes in terms of technical success (76\% vs. $77 \%$ ), clinical success ( $82 \%$ vs. $85 \%$ ), and adverse events (6\% vs. 5\%) between ERCP and PTBD. Technical and clinical success rates of ERCP for biliary-enteric anastomotic stricture in this study were similar to those previously reported in a multicenter study of enteroscopy-assisted ERCP in patients with surgically altered GI anatomy [6]. In the present study, however, despite comparable success and adverse event rates, ERCP was superior to PTBD in terms of length of hospital stay ( 0.2 vs. 4.5 days), number of procedures (4.6 vs. 9.5), and time to stricture resolution (1 vs. 7 months). As Hammad et al. discussed [5], ERCP enables direct visualization of the anastomotic stricture as well as stricture resolution after dilation even at index ERCP.

Yousuke Nakai

ynakai-tky@umin.ac.jp

1 Department of Endoscopy and Endoscopic Surgery, Graduate School of Medicine, The University of Tokyo, 7-3-1 Hongo Bunkyo-ku, Tokyo 113-8655, Japan
Meanwhile, technical failure due to guidewire passage across the stricture was observed in six cases at index PTBD procedure, necessitating additional procedures. Furthermore, clinical success of the PTBD approach was evaluated under fluoroscopic guidance, not under direct visualization, which might increase the number of sessions in the PTBD group.

Despite the encouraging results reported in this study, there are several caveats regarding enteroscopy-assisted ERCP. First, the mean procedure time for the index ERCP was more than twice that of the index PTBD (110 vs. $50 \mathrm{~min}$ ) due to the long-limb anatomy. Second, long-term outcomes after stricture resolution are not fully reported. Although biliary stent or PTBD tube placement is usually maintained for 6-12 months for benign biliary strictures, the median treatment duration after ERCP was only 1 month in this study. Thus, the rate of stricture recurrence should be further evaluated in long-term follow-up. Third, while repeat PTBD was performed in two-thirds of failed PTBD cases, 10 out of 11 failed ERCP cases underwent rescue PTBD, suggesting some bias in treatment selection. Finally, enteroscopy-assisted ERCP was not compared to endoscopic ultrasound (EUS)-guided biliary interventions. Recent reports have demonstrated technical success rates of $>95 \%$ with the latter technique [7]. The EUS-guided approach, like the ERCP approach, provides internal drainage but does not require deep endoscope insertion into the biliary-enteric anastomosis. Accordingly, the EUS approach has a shorter procedure time and, long-term, stent exchange can be more easily performed. An international multicenter comparative study [8], which included both malignant and benign biliary obstruction, revealed the superiority of EUS-guided biliary drainage in terms of technical and clinical success and procedure time. Meanwhile, enteroscopy-assisted ERCP provided lower adverse event rate and shorter length of hospital stay.

In summary, enteroscopy-assisted ERCP may be considered first-line therapy for the management of biliaryenteric anastomotic strictures in patients with a long-limb 
Roux-en-Y reconstruction. Nevertheless, technical failure occurs in $\sim 20-30 \%$ due to the altered anatomy. PTBD and EUS-guided interventions may be considered useful rescue procedures. Thus, there are at least three complementary procedures that may be employed depending upon patient anatomy and the local expertise. Since EUS-guided interventions are increasingly reported, a prospective study should be conducted, comparing enteroscopy-assisted ERCP to EUSguided interventions.

\section{References}

1. Born P, Rosch T, Bruhl K, et al. Long-term results of endoscopic and percutaneous transhepatic treatment of benign biliary strictures. Endoscopy. 1999;31:725-731.

2. Kaffes AJ. Management of benign biliary strictures: current status and perspective. J Hepato-Biliary-Pancreat Sci. 2015;22:657-663.

3. Moreels TG. Update in enteroscopy: new devices and new indications. Dig Endosc. 2018;30:174-181.

4. Yamada A, Kogure H, Nakai Y, et al. Performance of a new shorttype double-balloon endoscope with advanced force transmission and adaptive bending for pancreaticobiliary intervention in patients with surgically altered anatomy: a propensity-matched analysis. Dig Endosc. 2019;31:86-93.

5. Hammad H, Brauer BC, Smolkin M, Ryu R, Obuch J, Shah RJ. Treating biliary-enteric anastomotic strictures with enteroscopyERCP requires fewer procedures than percutaneous transhepatic biliary drains. Dig Dis Sci. (Epub ahead of print). https://doi. org/10.1007/s10620-019-05670-y.

6. Shah RJ, Smolkin M, Yen R, et al. A multicenter, US experience of single-balloon, double-balloon, and rotational overtubeassisted enteroscopy ERCP in patients with surgically altered pancreaticobiliary anatomy (with video). Gastrointest Endosc. 2013;77:593-600.

7. Nakai Y, Kogure H, Isayama H, Koike K. Endoscopic ultrasoundguided biliary drainage for benign biliary diseases. Clin Endosc. 2019;52:212-219.

8. Khashab MA, El Zein MH, Sharzehi K, et al. EUS-guided biliary drainage or enteroscopy-assisted ERCP in patients with surgical anatomy and biliary obstruction: an international comparative study. Endosc Int Open. 2016;4:E1322-e1327.

Publisher's Note Springer Nature remains neutral with regard to jurisdictional claims in published maps and institutional affiliations. 